\title{
LE CHANGEMENT CLIMATIQUE MISE EN ÉVIDENCE, DÉNI ET DÉFIS
}

\author{
GUY MUNHOVEN
}

RÉsumÉ. Depuis le début de l'ère industrielle, la température de surface de la Terre a augmenté de plus d'un degré Celsius en moyenne. Notre compréhension scientifique du système climatique terrestre, qui s'est construite au cours des deux siècles passés, ne laisse que très peu de doute : ce réchauffement n'est pas d'origine naturelle, il est lié aux émissions de gaz à effet de serre, surtout de $\mathrm{CO}_{2}$, mais aussi de $\mathrm{CH}_{4}$, de $\mathrm{N}_{2} \mathrm{O}$ ou de gaz halogènes par l'activité humaine. Dans cette contribution, je vous présenterai brièvement les principaux indicateurs montrant que le climat subit effectivement un changement important actuellement, que ce changement est lié à un renforcement de l'effet de serre naturel, et que ce sont les gaz à effet de serre émis par l'activité humaine qui sont à l'origine de ce bouleversement. Je passerai en revue quelques étapes et tournants importants de l'histoire du développement des sciences climatiques. Partant d'une série d'arguments "climato-sceptiques" courants, je vous présenterai les développements scientifiques récents

Enfin nous allons examiner les complications liés au transfert et à la transposition des connaissances scientifiques en des mesures politiques adéquates pour mitiger le changement climatique ou pour s'y adapter, les défis et pièges potentiels liés à la communication de la science climatique aux dirigeants politiques.

\section{INTRODUCTION}

En 1957, Roger Revelle et Hans Suess [1] déclarent que :

« Ainsi, les êtres humains réalisent actuellement une expérience géophysique à grande échelle d'un type qui n'aurait pas pu se faire dans le passé ni être reproduit à l'avenir. Endéans quelques siècles, nous retournons dans l'atmosphère et les océans le carbone organique concentré qui s'est accumulé dans les roches sédimentaires pendant des centaines de millions d'années. Cette expérience, si elle est documentée de manière adéquate, peut donner

Date: 28 novembre 2019 .

L'auteur est Chercheur Qualifié au Fonds de la Recherche Scientifique-FNRS. 
une idée approfondie des processus déterminant le temps et le climat. »

Cette étude, avec celles de Gilbert N. Plass [2, 3] publiées une année plus tôt, marque un tournant dans l'acceptation de la théorie reliant des variations du climat terrestre aux variations des concentrations de $\mathrm{CO}_{2}$ dans l'atmosphère. Cette théorie avait été publiée en 1896 par Svante Arrhenius [4, réfutée en 1900 déjà par Knut ÅngStröm [5] - de manière injustifiée comme il s'est avéré plus tard.

La déclaration de REVELle et SuESS est devenue programme et soixante ans plus tard, la connaissance des «processus de temps et de climat » a effectivement fait des progrès immenses et continue à en faire.

Je vous propose de passer en revue quelques étapes importantes de la découverte scientifique du changement climatique, le scepticisme qui a mis à l'épreuve ces découvertes et les développements théoriques associés et la mutation de ce scepticisme en déni. Je confronterai quelques arguments souvent avancés pour dénier, minimiser ou relativiser l'importance du changement climatique actuel aux résultats d'études récentes et de données publiquement accessibles. Enfin, nous allons examiner comment la science peut contribuer à mieux cerner le défi que représente le changement climatique pour la société.

\section{Mise en Évidence : DÉteCtion et Attribution}

La mise en évidence du changement climatique actuel comprend deux grands volets : celui de la détection (le climat change-t-il vraiment?) et l'attribution (si oui, quelles en sont les causes?). Les principaux indicateurs du changement climatique actuel sont ([6, FAQ 2.1])

- augmentation de la température des eaux de surface des océans;

- augmentation de la température de l'air au-dessus des terres émergées et des océans;

- augmentation de la température de l'air dans la troposphère;

- augmentation de l'humidité relative dans la troposphère;

- accumulation de chaleur dans les océans;

- élévation du niveau de la mer, dont la majeure partie résulte actuellement de l'expansion de volume suite à l'augmentation de température qui accompagne l'accumulation de chaleur (donc lié au précédent);

- diminution de la couverture neigeuse;

1. «Thus human beings are now carrying out a large scale geophysical experiment of a kind that could not have happened in the past nor be reproduced in the future. Within a few centuries we are returning to the atmosphere and oceans the concentrated organic carbon stored in sedimentary rocks over hundreds of millions of years. This experiment, if adequately documented, may yield a far-reaching insight into the processes determining weather and climate. » 
— diminution de l'étendue des glaces de mer et du volume des glaciers continentaux.

Ces observations indiquent aussi que le changement actuellement observé est fondamentalement un réchauffement global auquel sont associés d'autres changements climatiques.

A priori, ces changements pourraient être liés à divers facteurs de forçage, comme des variations de l'insolation, des changements dans la fréquence ou nature d'éruptions volcaniques (via les aérosols envoyés dans l'atmosphère au cours de ces éruptions) ou éventuellement des oscillations internes au système. Il y a cependant une multitude d'indices montrant que le réchauffement actuellement observé est principalement lié à un renforcement de l'effet de serre :

— réchauffement plus important de nuit que de jour [7]

— réchauffement plus important sur les continents qu'au dessus des océans

- réchauffement de la troposphère accompagnée d'un refroidissement dans la stratosphère [8, 9], la mésosphère [10, 11] et dans l'atmosphère supérieure en général [12];

- augmentation du rayonnement à grande longueurs d'onde (infrarouge) vers le bas [13] et diminution vers le haut [14]

- augmentation des concentrations de $\mathrm{CO}_{2}$ et autres gaz à effet de serre $\left(\mathrm{CH}_{4}, \mathrm{~N}_{2} \mathrm{O}\right.$, ozone troposphérique, gaz halogènes) dans l'atmosphère.

La croissance des concentrations des gaz à effet de serre dans l'atmosphère pourrait, a priori encore une fois, résulter d'une réorganisation des sources et puits naturels, surtout en ce qui concerne le $\mathrm{CO}_{2}$ et le $\mathrm{CH}_{4}$. Il y a cependant de nouveau plusieurs indices concordant avec une origine anthropogénique:

- augmentation de la concentration de $\mathrm{CO}_{2}$ dans l'atmosphère

- évolution des émissions de $\mathrm{CO}_{2}$ liées à l'utilisation de combustibles fossiles, de la production de ciments, à la déforestation et au changement d'affectation des sols;

- diminution conjointe du rapport ${ }^{13} \mathrm{C} /{ }^{12} \mathrm{C}$ (mesures directes sur le $\mathrm{CO}_{2}$ atmosphériques, indirectes dans les cernes d'arbres et dans les coraux);

- diminution conjointe de la fraction de radiocarbone, ${ }^{14} \mathrm{C}$ (essentiellement mesures indirectes en raison de la perturbation trop importante du bilan $\mathrm{du}{ }^{14} \mathrm{C}$ dans l'atmosphère suite aux essais nucléaires aériens pendant les années '50 et le début des années '60);

- diminution conjointe de la concentration d'oxygène dans l'atmosphère ;

- concentrations des autres gaz à effet de serre naturels dans l'atmosphère largement supérieures à leurs concentrations naturelles 
au cours des dernières centaines de milliers d'années; les gaz halogènes, comme les $\mathrm{CFC}$, sont entièrement d'origine humaine;

- contribution trop faible des forçages naturels (activité du Soleil, éruptions volcaniques, ...) dans le bilan d'énergie de la Terre.

La diminution du rapport ${ }^{13} \mathrm{C} /{ }^{12} \mathrm{C} d u \mathrm{CO}_{2}$ atmosphérique révèle une origine organique. ${ }^{2}$ Deux sources pour le surplus de $\mathrm{CO}_{2}$ sont dès lors possibles : du carbone biosphérique ou du carbone provenant de ressources fossiles, qui sont aussi d'origine organique. L'évolution conjointe de la fraction de radiocarbone dans le $\mathrm{CO}_{2}$ atmosphérique indique cependant que la source du $\mathrm{CO}_{2}$ doit être en très grande partie fossile, c'est-à-dire, dépourvue de ${ }^{14} \mathrm{C}$. Le radiocarbone a une demi-vie de $5730 \mathrm{ans}$; du carbone en provenance de la biosphère (végétation ou sols) en contient, mais non pas des combustibles fossiles formés il y des dizaines ou des centaines de millions d'années.

Les premiers relevés systématiques de l'évolution de la concentration d'oxygène dans l'atmosphère (dérivés du rapport $\mathrm{O}_{2} / \mathrm{N}_{2}$, mesuré par une technique interférométrique, qui permet d'atteindre une précision de \pm 4 parts par million) ont été effectuées à partir de 1989 à La Jolla (Californie) par RALPH KeELing [15]. L'évolution de la concentration d'oxygène dans l'atmosphère se caractérise par un cycle saisonnier résultant de l'échange avec la biosphère (photosynthèse et respiration) ainsi qu'à l'échange air-mer, superposé à une tendance décroissante. La décroissance mesurée est légèrement plus faible que celle calculée à partir des données sur l'utilisation de combustibles fossiles [16], reflétant le fait que la biosphère terrestre et les océans agissent comme des puits pour le $\mathrm{CO}_{2}$ d'origine anthropique. Or l'assimilation de $\mathrm{CO}_{2}$ par photosynthèse agit évidemment comme source d'oxygène pour l'atmosphère.

\section{Du SCEPTICISME AU DÉNi}

L'effet de serre joue donc un rôle central pour expliquer la température à la surface de la Terre. Passons donc en revue quelques étapes importantes et moments-clé de l'histoire de sa découverte. Pour plus d'informations et de détails, je vous recommande l'historique assez complet et accessible publié par WEART [17] (voir aussi le site web https://history.aip.org/climate qui accompagne le livre). Une autre perspective historique est aussi fournie dans le premier chapitre $\mathrm{du} 4^{\mathrm{e}}$ Rapport d'évaluation du Giec [18]. Le New York Times MAGAZINE a publié une enquête intitulée «Losing Earth : The Decade We

2. La matière organique produite par photosynthèse présente des rapports ${ }^{13} \mathrm{C} /{ }^{12} \mathrm{C}$ de l'ordre de 20 à 25 pour mille plus bas que le $\mathrm{CO}_{2}$ consommé. 
Almost Stopped Climate Change », très détaillée et orientée grand public qui couvre plus spécialement les événements importants des années 1979 à 1989.3

3.1. Petit historique de la théorie de l'effet de serre. L'histoire de l'effet de serre remonte près de deux siècles en arrière, voire un peu plus, si on inclut la découverte du rayonnement infrarouge par l'astronome WiLliam HeRSCHEL en 1800, rayonnement qualifié d' « obscur » ou « rayons calorifiques » à l'époque. | [ En 1824, Joseph FouRIER [19, p. 155] la décrivait pour la première fois dans son mémoire «Remarques générales sur les températures du globe terrestre et des espaces planétaires $\gg$ :

«[L]a température peut être augmentée par l'interposition de l'atmosphère, parce que la chaleur trouve moins d'obstacle pour pénétrer l'air, étant à l'état de lumière, qu'elle n'en trouve pour repasser dans l'air lorsqu'elle est convertie en chaleur obscure. »

Le phénomène, décrit encore en termes assez qualitatifs par FOURIER, était élucidé vers 1860 par John TyNDALL, qui a démontré de manière expérimentale, que certains gaz, comme le dioxyde de carbone et le méthane, ou encore la vapeur d'eau, pouvaient absorber les rayons calorifiques, contrairement à l'oxygène, l'azote ou l'hydrogène [20]. Il conclût que la vapeur d'eau présentait la capacité d'absorption la plus importante parmi les gaz étudiés et que c'était donc elle qui exerçait le contrôle principal sur la température de l'air.

La première prédiction quantitative liée à une perturbation de l'effet de serre naturel a été publiée en 1896 par Svante Arrhenius [4], prédisant qu'un doublement de $\mathrm{CO}_{2}$ dans l'atmosphère devait conduire à un réchauffement global de 5 ou $6^{\circ} \mathrm{C}$. Le travail d'ARRHEniUs a été accueilli avec beaucoup de scepticisme et rapidement remis en question par KNUT ÅNGSTRÖM [5], qui argumentait-résultats expérimentaux à l'appui-que les bandes d'absorption du $\mathrm{CO}_{2}$ étaient déjà saturées à la teneur en $\mathrm{CO}_{2}$ atmosphérique de l'époque, c'est-à-dire, qu'en pratique, l'atmosphère était déjà opaque au rayonnement infrarouge. Ajouter du $\mathrm{CO}_{2}$ ne devrait donc pas modifier son pouvoir d'absorption.

La chambre d'écho médiatique fonctionnait aussi déjà au début du XX ${ }^{\mathrm{e}}$ siècle : dans le Monthly Weather Review de juin 1901, une critique de l'article d'ÅnGSTRÖM inclut la mise en garde suivante : «Les géologues qui ont adopté les vues d'Arrhenius devraient se rappeler l'échec évident de son hypothèse à la lumière des connaissances

3. Consultable sous https://www.nytimes.com/interactive/2018/08/01/ magazine/climate-change-losing-earth.html; le quotidien suisse LE TEMPS en a fait un résumé en français (https://www.letemps.ch/sciences/ avons-perdu-combat-contre-changement-climatique).

4. La dénomination 《infrarouge » n'apparaîtra que beaucoup plus tard. Même TyNDALL ne l'utilisait pas encore dans son article publié en 1861. 
actuelles du pouvoir d'absorption du dioxyde de carbone. $\gg 5^{5}$ Il faut savoir que l'intérêt premier d'ARRHENIUS, comme il le précise dans son article, était de fournir une explication pour les climats froids des âges glaciaires et pour les climats chauds du Tertiaire aux géologues.

Cependant, l'expérience d'ÅnGSTRÖM ne permettait pas de soutenir sa conclusion finale, qui soutenait que, étant donné que les bandes d'absorption du $\mathrm{CO}_{2}$ étaient déjà à saturation et qu'il y avait un recouvrement important de ces bandes avec celles de la vapeur d'eau, une augmentation ou une diminution des concentrations de $\mathrm{CO}_{2}$ d'un facteur deux n'aurait qu'un effet négligeable sur les températures de surface de la Terre. En effet, le comportement radiatif du $\mathrm{CO}_{2}$ dans la colonne atmosphérique ne peut pas être simulé de manière réaliste à l'aide d'un tube cylindrique, qui contient la quantité équivalente sous forme pure, pour la simple raison que la température décroît avec l'altitude dans la troposphère, qui contient la majeure partie de la masse de l'atmosphère. Les implications de ce profil de température sur la bilan radiatif de l'atmosphère étaient pourtant connues à l'époque. En tout cas, le météorologue suédois NiLs EKHOLm écrivait en 1901 que [21, p. 20]

« [...] le rayonnement de la [T]erre dans l'espace ne provient pas directement de la surface, mais en moyenne d'une couche de l'atmosphère à une altitude considérablement au-dessus du niveau de la mer. L'altitude de cette couche dépend des propriétés thermiques de l'atmosphère et variera en fonction de ces propriétés. Plus le pouvoir absorbant de l'air pour le rayonnement thermique émis par la surface est important, plus cette couche sera élevée. Mais plus la couche est élevée, plus sa température est basse par rapport à celle du sol; et comme le rayonnement de la couche dans l'espace est d'autant plus faible que sa température est basse, il s'ensuit que le sol sera d'autant plus chaud que la couche rayonnante sera haute. $\gg 6$

5. "The geologists who have adopted Arrhenius's views should recall that his hypothesis evidently fails in the light of present knowledge of the absorptive powers of carbon dioxid [sic]. »

6. «[...] the radiation from the earth into space does not go on directly from the ground, but on the average from a layer of the atmosphere having a considerable height above sea-level. The height of that layer depends on the thermal quality of the atmosphere, and will vary with that quality. The greater is the absorbing power of the air for heat rays emitted from the ground, the higher will that layer be. But the higher the layer, the lower is its temperature relatively to that of the ground; and as the radiation from the layer into space is the less the lower its temperature is, it follows that the ground will be hotter the higher the radiating layer is. » 
Cette description est étonnamment proche de notre compréhension actuelle du fonctionnement de l'effet de serre (voir, p. ex., [22]). AnGSTRÖM était-il au courant de cette idée? Éventuellement, oui. Tout d'abord, il reconnaît explicitement cette lacune de connaissance [5, p. 722] : «Il n'est pas encore possible d'évaluer l'importance de l'absorption par le dioxyde de carbone dans les parties plus hautes de l'atmosphère terrestre. » $]^{7}$ L'article d'EKHOLM [21] est, d'après son préambule, la «traduction revue et étendue » d'un article publié antérieurement en suédois [23] et qui était cité par Ångström [5, p. 731]! Cependant, à ma meilleure connaissance, le passage cité ci-dessus ne figure pas dans la version d'origine en suédois [23]. Il est donc bien possible qu'ÅngSTRÖM n'ait pas eu connaissance de cette idée, ou, qu'il en avait connaissance, mais qu'il préférait ne pas en tenir compte, pour une raison non spécifiée.

Ce n'est qu'après la seconde guerre mondiale, avec les avancées dans l'observation de la haute atmosphère, qu'il a été possible d'établir que les bandes d'absorption du rayonnement infrarouge par le $\mathrm{CO}_{2}$ ne sont effectivement pas saturées à haute altitude, ce qui, en raison des concentrations très faibles en vapeur d'eau à ces mêmes altitudes, invalidait donc la réfutation catégorique d'ÅngSTRÖM [5].

Toujours est-il que l'argument de saturation des bandes d'absorption du $\mathrm{CO}_{2}$ a temporairement mis fin à la théorie climat-dioxyde-decarbone-atmosphérique proposée par Svante ARRHENIUs et raffinée par NiLs EKHOLM. La théorie n'est pas tombée dans l'oubli pour autant, mais pendant plus de cinquante ans, il n'y aura que quelques études isolées qui y référeront de manière positive, comme, p. ex. celle de Callendar [24]. Guy S. Callendar était un ingénieur hydraulique (steam engineer) anglais et météorologue amateur pendant son temps libre. Par intérêt personnel, il avait compilé des relevés de température de deux cents stations météorologiques à travers le monde pour construire ainsi une toute première reconstruction de l'évolution de la température moyenne de la surface terrestre. Il trouva que cette température avait augmenté à raison d'environ $0,05^{\circ} \mathrm{C}$ /décennie entre 1880 et 1935. Il estime aussi que la concentration de $\mathrm{CO}_{2}$ dans l'atmosphère avait augmenté d'environ $6 \%$ depuis le début du $\mathrm{Xx}^{\mathrm{e}}$ siècle et il montra qu'un tel accroissement avait produit un réchauffement à raison d'environ $0,03^{\circ} \mathrm{C} /$ décennie. Il en déduisit que l'accumulation de $\mathrm{CO}_{2}$ dans l'atmosphère résultant de l'utilisation de combustibles fossiles jouait un rôle considérable dans le réchauffement qu'il avait observé. La communauté scientifique avait accueilli les résultats de CALLENDAR avec une certaine bienveillance, mais le doute quant à la signification de l'augmentation de $\mathrm{CO}_{2}$ dans l'atmosphère qu'il avait calculé l'emportait à

7. «Wie gross die Kohlensäureabsorption in den höheren Teilen der Erdatmosphäre ist, lässt sich noch nicht beurteilen. » 
la fin. On n'avait que peu de confiance en la validité des mesures de $\mathrm{CO}_{2}$ de la fin du XIX ${ }^{\mathrm{e}}$ siècle.

Il fallait attendre les années ' $50 \mathrm{du} \mathrm{XX}^{\mathrm{e}}$ siècle et les travaux de GILBERT N. Plass [2, 3] pour sortir la théorie de l'effet de serre de sa léthargie et la réhabiliter. PLASS faisait appel à des résultats de mesures d'absorption de dioxyde de carbone dans l'infrarouge dans la bande à $15 \mu \mathrm{m}$ publiés peu avant. Il trouvait qu'un doublement de la concentration de $\mathrm{CO}_{2}$ conduirait à une augmentation de la température de surface de $3,5^{\circ} \mathrm{C}$ en moyenne, tandis qu'une réduction de moitié conduirait à un refroidissement de $3,8^{\circ} \mathrm{C}$. Il conclut que [2, p. 320] :

« Les calculs de rayonnement prédisent clairement un changement de température précis pour chaque variation de la quantité de $\mathrm{CO}_{2}$ dans l'atmosphère. Ces changements de température sont suffisamment importants pour influencer le climat de manière appréciable. ${ }^{8}$

Les résultats et la conclusion à laquelle PLASS était arrivé, rencontraient aussi quelques réactions sceptiques, et pour de bonnes raisons. Ainsi, KAPlan [25] calcula que, si la couverture des nuages est prise en compte (que PLASS n'avait que brièvement évoquée), la diminution de température pour une réduction de $\mathrm{CO}_{2}$ de moitié serait deux à trois fois plus faible. MÖLler [26] reprit les travaux de PLASS et de KAPLAN afin d'analyser comment les résultats seraient modifiés si la rétroaction de la vapeur d'eau était aussi considérée. Il se retrouva avec un bilan d'énergie à la surface terrestre qui rend les résultats de ses calculs très erratiques : des changements de l'ordre de $1 \%$ de la couverture nuageuse ou de 3\% de l'humidité (nettement en-dessous du seuil de précision des observations) pouvaient contre-balancer les effets d'un doublement du $\mathrm{CO}_{2}$. Il conclut qu' « [e]n raison de ces valeurs, toute la théorie des changements climatiques dus aux variations de $\mathrm{CO}_{2}$ devient contestable. $\gg 9^{9}$ Mais, il ne condamna pas la théorie pour autant. En bon sceptique, il terminait son article en soulignant que le bilan d'énergie de surface adopté dans sa démarche était incomplet, car il avait négligé les termes liés aux échanges de chaleur sensible et latente, et que des variations d'un des termes du bilan engendreraient des variations de tous les autres. Les effets de telles variations conjointes devraient faire l'objet d'une étude ultérieure...

Cette étude ultérieure était publiée quatre ans plus tard par SYUkuro Manabe et Richard T. Wetherald [27]. Ce papier marque

8. "The radiation calculations predict a definite temperature change for every variation in $\mathrm{CO}_{2}$ amount in the atmosphere. These temperature changes are sufficiently large to have an appreciable influence on the climate. »

9. "Because of these values the entire theory of climatic changes by $\mathrm{CO}_{2}$ variations is becoming questionable.» 
l'une des percées les plus importantes - peut-être même la percée définitive - pour notre compréhension des mécanismes physiques qui contrôlent le fonctionnement du système climatique. MAnABE et WetheRALD prouvent (1) que la suspicion de MöLlER [26] était fondée, et (2) que bilan radiatif à la surface de la Terre était insuffisant pour évaluer des changements de température liés à des variations de $\mathrm{CO}_{2}$, mais qu'il fallait aussi tenir compte du bilan d'énergie de toute la colonne atmosphérique. 10

Entre-temps, Charles D. KeEling commence son programme de mesure du $\mathrm{CO}_{2}$ atmosphérique : en 1957, dans le cadre de l'Année Géophysique Internationale, au Pôle Sud, l'année suivante à l'observatoire de Mauna Loa (Hawaï). Après deux ans de mesures, KeELing peut déjà tirer les premières conclusions [28] : la concentration de $\mathrm{CO}_{2}$ suit un cycle saisonnier bien marqué, ainsi qu'une tendance à la croissance, estimée à environ 1 ppm/an (1 ppm est égal à une part par million (en volume)). Au fil des années, la tendance à la croissance du $\mathrm{CO}_{2}$ dans l'atmosphère se confirme et s'accélère (voir figure 1). D'autres stations de mesure s'ajoutent au fil du temps pour compléter l'image globale. Le cycle saisonnier, lié avant tout aux échanges de $\mathrm{CO}_{2}$ entre la biosphère par photosynthèse au printemps et en été, et respiration en automne et en hiver est plus marqué en hémisphère Nord qu'en hémisphère Sud. La tendance à la croissance est très semblable en toutes les stations, légèrement plus lente au Pôle Sud qu'en hémisphère Nord. En moyenne décennale, le taux de croissance à Mauna Loa passe de 8,9 ppm/décennie pour la décennie 1961-1970 à 13,5 ppm/décennie pour 1971-1980, puis à 16,4 ppm/décennie pour 1981-1990. Pour la décennie en cours (20112020), le taux de croissance peut être estimé à 24,8 ppm/décennie sur base des données disponibles jusque juin 2019.

Vers la fin des années '60, la théorie de l'effet de serre était donc finalement établie. Elle pouvait expliquer les processus en œuvre dans l'atmosphère et permettait de faire des prédictions vérifiables, comme, p. ex., le refroidissement stratosphérique lié à un doublement de $\mathrm{CO}_{2}$, mis en évidence par Manabe \& Wetherald [27]. Le scepticisme, cette éternelle remise en question des connaissances scientifiques, même s'il a contribué à freiner le développement et l'acceptation de la théorie, a aussi contribué à sa consolidation.

Maintenant que le rôle du $\mathrm{CO}_{2}$ pour l'effet de serre était reconnu et que l'évolution du $\mathrm{CO}_{2}$ atmosphérique documentée par KeELing ne montrait pas de signe de ralentissement, les inquiétudes concernant les conséquences à long terme sur le climat et l'impact sur l'humanité ont

10. Cette merveilleuse publication claire et complète mérite d'être lue encore plus de cinquante ans après sa publication. Elle est disponible en accès ouvert dans les archives de l'Americal Meteorological Society. 


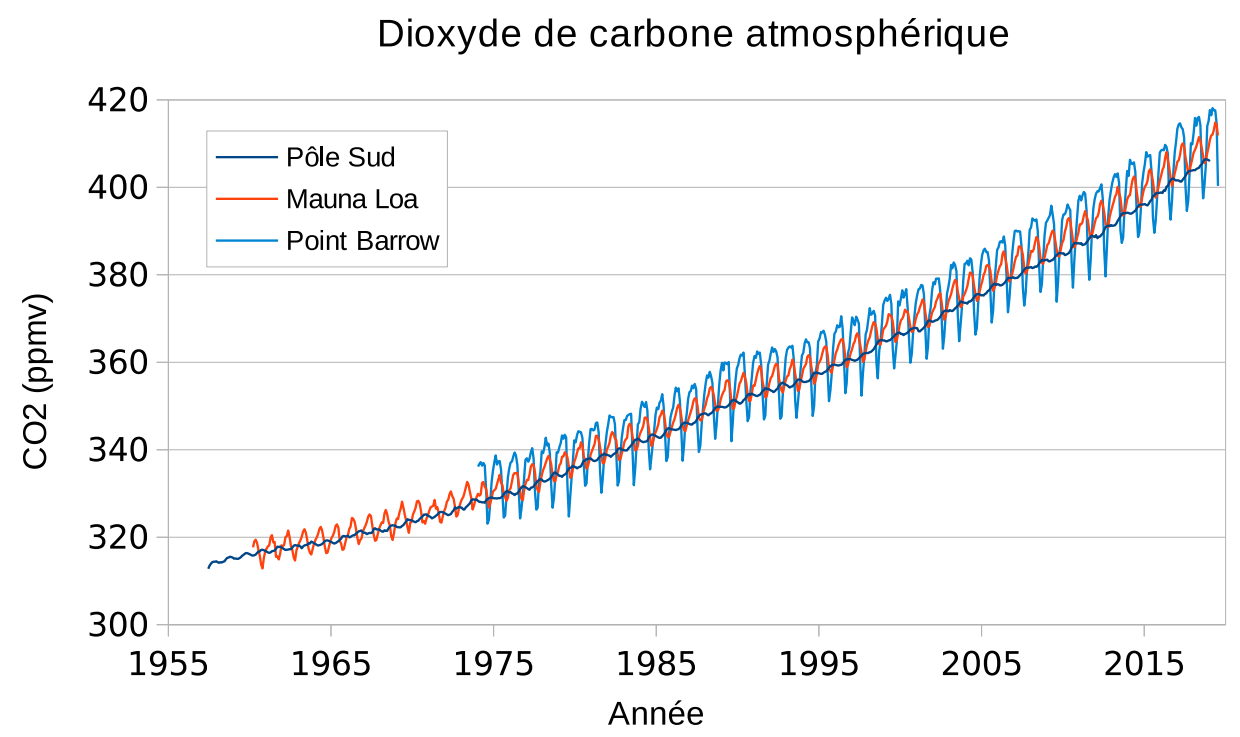

Figure 1. Évolution des concentrations de $\mathrm{CO}_{2}$ dans l'atmosphère au Pôle Sud, à Mauna Loa (Hawaï) et à Point Barrow (Alaska), d'après les données du SCRIPPS CO2 Program. Les concentrations de $\mathrm{CO}_{2}$ sont exprimées en parts par million en volume (ppmv).

commencé à devenir pressantes. Mais avec la prise de conscience, autant par le public que par les milieux politiques, de la réalité du danger que des émissions non contrôlées de $\mathrm{CO}_{2}$ dans l'atmosphère allait poser, et que des mesures effectives pour combattre ou du moins atténuer les effets allaient demander une réduction substantielle de l'utilisation de combustibles fossiles, une opposition puissante allait se former : il y avait maintenant des intérêts économiques en jeu. Au courant des années'90, les puissants lobbys des industries du pétrole, du gaz et du charbon, ainsi que des think tanks spécialement fondés à cette fin, organisent des compagnes savamment orchestrées pour remettre en question la crédibilité de la théorie, et si cela s'avérait utile ou prometteur, aussi celle de ses supporters, sous un couvert de scepticisme. Comme des mémos internes des compagnies et organisations impliquées montrent, le but déclaré était de semer le doute (en insistant sur les incertitudes, en contestant la validité de certaines données et de leur interprétation, ...), de relativiser ou de minimiser l'influence du $\mathrm{CO}_{2}$ ou encore en remettant en question le rôle de l'Homme dans les perturbations observées. La stratégie n'avait clairement plus rien à voir avec l'objectif intrinsèque de toute recherche scientifique, à savoir l'amélioration des connaissances, mais surtout de discréditer et de remettre en question le savoir établi, de fabriquer et d'alimenter une controverse artificielle, ou alors de créer des attentes impossibles à remplir par la recherche. 
C'est ce qui distingue le scepticisme du déni [29, 30] et je vais utiliser ce qualificatif par la suite. Pour plus d'informations sur cette partie déplaisante des sciences climatiques, je réfère de nouveau au livre de WEART [17, qui en couvre quelques épisodes (le site qui accompagne le livre en fournit d'autres) ainsi qu'à l'analyse publiée par la UNION OF CONCERNED SCIENTISTS, étayée de manière convaincante par des document originaux [31].

3.2. Arguments, affirmations et contre-vérités. Je vais me servir de quelques affirmations couramment avancées pour mettre en question la réalité du changement climatique, du rôle du $\mathrm{CO}_{2}$, etc. pour rapporter quelques avancées récentes dans le domaine.

3.2.1. Le climat a toujours changé ... Le climat a bien sûr toujours changé. La Terre semble même avoir été complètement gelée plusieurs fois-les fameux épisodes « Terre boule de neige »-dont le dernier s'est terminé il y a 635 millions d'années environ. Au début de l'Éocène, il y a un peu plus de cinquante millions d'années, des alligators et tortures géantes peuplaient l'Arctique canadien (voir p. ex., [32]).

$\mathrm{Au}$ passé géologique plus récent, le Pléistocène, qui s'étend d'il y a 2,58 millions à 11700 années avant le présent, a été rythmé par l'alternance entre périodes glaciaires (froides) et interglaciaires (chaudes), avec une périodicité de l'ordre de 41000 ans au début, puis de 100000 ans vers la fin.

Les périodes chaudes du passé géologique sont souvent utilisées comme analogues pour illustrer les conséquences potentielles d'un réchauffement soutenu au cours des prochaines décennies et siècles. La dernière période interglaciaire, l'Eémien (il y a 130 à 119 milliers d'années) fait partie de ces analogues. L'Eémien était caractérisé par une température moyenne de $3^{\circ} \mathrm{C}$ plus élevée qu'à l'ère préindustrielle, une différence qui se situe au milieu de la fourchette des valeurs de réchauffement les plus probables en cas d'un doublement de $\mathrm{CO}_{2}$ dans l'atmosphère, qui est de 1,5 à $4,5^{\circ} \mathrm{C}$. La plupart du temps, le niveau de la mer était $3 \mathrm{~m}$ plus élevé qu'actuellement. Vers la fin, il montait cependant à $6 \mathrm{~m}$, puis temporairement même à $9 \mathrm{~m}$ au-dessus du niveau [33]. Il convient de noter que le réchauffement n'était pas le résultat d'un taux de $\mathrm{CO}_{2}$ élevé dans l'atmosphère (le taux était sensiblement le même qu'à l'ère préindustrielle), mais à une distribution d'insolation très différente de l'actuelle : l'orbite de la Terre était plus excentrique, l'été en hémisphère Nord proche du périhélie et l'inclinaison de l'axe de rotation du globe plus inclinée qu'aujourd'hui. L'analogue n'est donc pas parfait, mais illustre bien les répercussions d'un réchauffement de l'ordre de grandeur de celui attendu en cas de doublement de $\mathrm{CO}_{2}$.

Au cours du passé historique, le climat n'est pas resté stable non plus et a connu des épisodes froids et chauds. Ainsi, l'une des objections les plus souvent avancées, visant à minimiser le rôle de l'activité humaine 
pour le changement climatique actuel, soutient que le réchauffement actuel fait simplement partie de l'évolution cyclique du climat. Le PETIT ÂGE GLACIAIRE et l'OPTIMUM CLIMATIQUE MÉDIÉVAL seraient les expressions extrêmes les plus récentes de ce cycle, auxquels s'ajoutent une autre période plus froide appelée Dark Ages Cold Period ou Late Antique Little Ice Age (de 400 à 800 ap. J.-C. environ) et l'OPTIMUM CLIMATIQUE ROMAin (300 av. à 550 apr. J.-C.).

Il y avait depuis longtemps des doutes quant à l'étendue géographique et la synchronicité de changements liés à ces différents épisodes. Ils ont essentiellement été mis en évidence et défini à partir d'observations en Europe et en Amérique du Nord (comme leurs noms le suggèrent déjà). Plusieurs travaux publiés en été 2019 ont confirmé cette vision.

Neukom ET AL. 34] ont montré qu'au cours des 2000 dernières années, la Terre n'a jamais connu de période froide ni chaude présentant la même cohérence spatio-temporelle globale que le réchauffement contemporain. De leur analyse de la base de données d'indicateurs climatiques la plus complète actuellement disponible pour les deux millénaires (qui comprend pas moins de sept cents enregistrements obtenus à partir de cernes d'arbres, de carottes de glace, de coraux, sédiments, ...), il ressort qu'il y a bien eu des changements climatiques marqués au cours des deux derniers millénaires. Ces épisodes ont toutefois gardé un caractère essentiellement régional, jamais global. Hormis le réchauffement de la fin du $\mathrm{XX}^{\mathrm{e}}$ siècle, les changements mis en évidence manquaient tous de cohérence spatiale. Ainsi, le PETIT ÂGE GLACIAIRE, qui s'étendait d'environ 1300 à 1850, comprend bien les périodes les plus froides du dernier millénaire. Une bonne partie de la planète a connu ses épisodes les plus froids au XIX ${ }^{\mathrm{e}}$ siècle, alors qu'ailleurs les températures les plus froides ont été retracées au XV (parties est et centrale de l'Océan Pacifique) et au XVII ${ }^{\mathrm{e}}$ siècles (nord-ouest de l'Europe et sud-est d'Amérique du Nord).

L'OPTIMUM CLIMATIQUE MÉDIÉVAL, qu'on associe avec une période plus chaude et sèche s'étendant de 800 à 1200 environ n'a pas été globale non plus. À peine $40 \%$ de la surface terrestre n'ont atteint leurs valeurs maximales simultanément.

Le réchauffement actuel est par contre de nature tout à fait différente. Il est caractérisé par une cohérence spatiale globale qu'aucun des autres épisodes de changement climatique analysés ne présente et touche déjà $98 \%$ de la surface du globe à la fin du $\mathrm{XX}^{\mathrm{e}}$ siècle; seules quelques parties d'Antarctique font exception.

Une telle cohérence spatiale peut difficilement s'expliquer par des causes naturelles. Le manque de cohérence spatiale dans les périodes plus marquées de changement climatique au cours des deux derniers millénaires peut par contre expliquer pourquoi ces périodes ne sont pas délimitées de manière cohérente dans le temps non plus. Ainsi, 
pour le début du PETIT ÂGE GLACIAIRE, on peut trouver des débuts situés entre 1250 et 1550 dans la littérature; le début de l'OPTIMUM CLIMATIQUE MÉDiÉVAL est parfois fixé en 900 au lieu de 800 et parfois elle s'étend jusqu'en 1400 au lieu de 1200.

Dans une étude conjointe, NEuKOM ET AL. [35] analysent la variabilité de la température moyenne globale, ce qui leur permet de considérer des échelles de temps plus courtes $(\simeq 20$ plutôt que $\simeq 50$ ans dans la première étude). Ils trouvent de nouveau que le réchauffement actuel est particulier : les tendances globales au réchauffement les plus importantes à l'échelle de vingt ans et plus sont observées pour la deuxième moitié du $\mathrm{XX}^{\mathrm{e}}$ siècle.

Une troisième étude [36] publiée à peu près en même temps souligne le rôle du volcanisme (plusieurs grandes éruptions en moins de trois décennies, dont celle du TAMBORA en 1815 et une d'origine inconnue en 1808/1809) dans la dernière phase du PETIT ÂGE GLACIAIRE. Le rôle du volcanisme avait déjà précédemment été mis en évidence pour son initiation [37].

3.2.2. Le réchauffement s'est arrêté! - Pause? Hiatus? Une affirmation assez populaire soutient que le réchauffement a cessé depuis 1998 (alternativement, depuis $(x-1998)$ années, où $x$ est l'année en cours), malgré que le taux de $\mathrm{CO}_{2}$ dans l'atmosphère ait continué d'augmenter depuis lors (et que le $\mathrm{CO}_{2}$ n'a donc aucune influence sur le climat...).

Confrontons donc, pour commencer, cette affirmation tout simplement aux données. Sur la figure 2, vous pouvez voir au panneau supérieur, l'évolution de la moyenne globale des anomalies de température de l'air à la surface terrestre (terres émergées et océans) de 1998 à 2014. J'ai choisi l'année 2014 comme date de fin pour la simple raison que cet argument était couramment utilisé en 2013 et 2014. Les exemples les plus anciens que j'ai pu retrouver datent de 2006. Le propos est pour le moins étrange, d'autant plus qu'il était même tenu par des scientifiques, qui auraient facilement pu confronter leurs dires à des données ; à partir 2014, il était même devenu franchement intenable, car les années 2005, 2007, 2009 et 2010 avaient toutes été plus chaudes que 1998, 2006 arrivant à égalité. La simple tendance linéaire superposée présente une pente de $0.097 \pm 0.041^{\circ} \mathrm{C}$ /décennie, qui est donc significativement positive.

Le choix de l'année 1998 comme début de la période d' « arrêt » dans cet « argument »n'est pas anodine : 1998 était influencé par l'épisode El Niño très intense de la saison 1997-98. El Niño, ce qui, en Espagnol, signifie en toute généralité 《le petit garçon », mais dans ce contexte particulier 《l'Enfant-Jésus » est le nom donné à un phénomène, qui touche l'Océan Pacifique de manière récurrente, tous les deux à sept ans environ. Lors d'un épisode El Niño, l'eau de surface de l'océan en Pacifique Est se réchauffe de manière anormale. Ce réchauffement démarre 


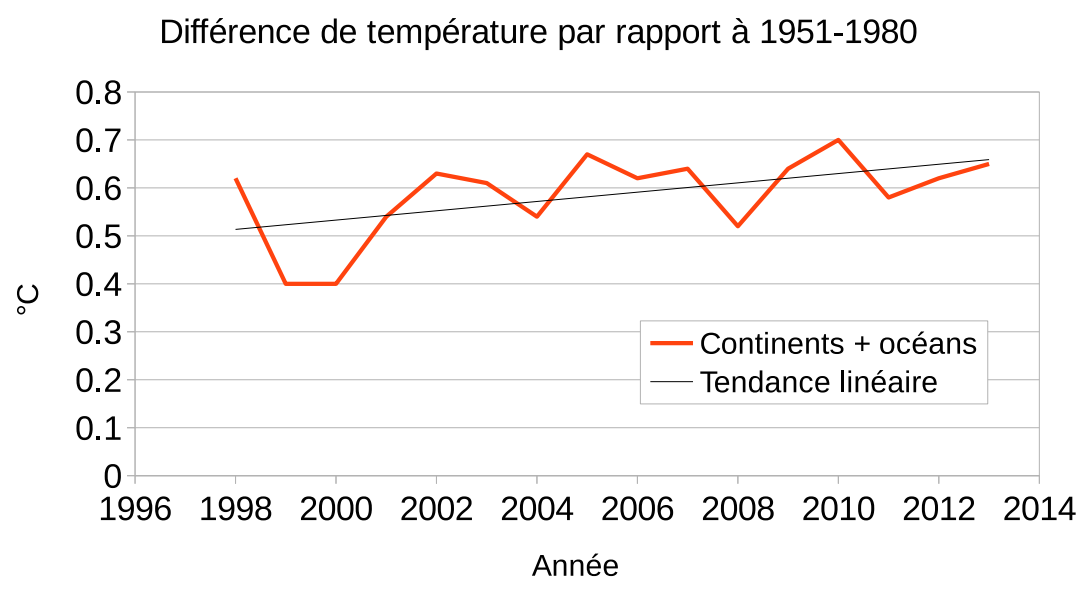

Différence de température par rapport à 1951-1980

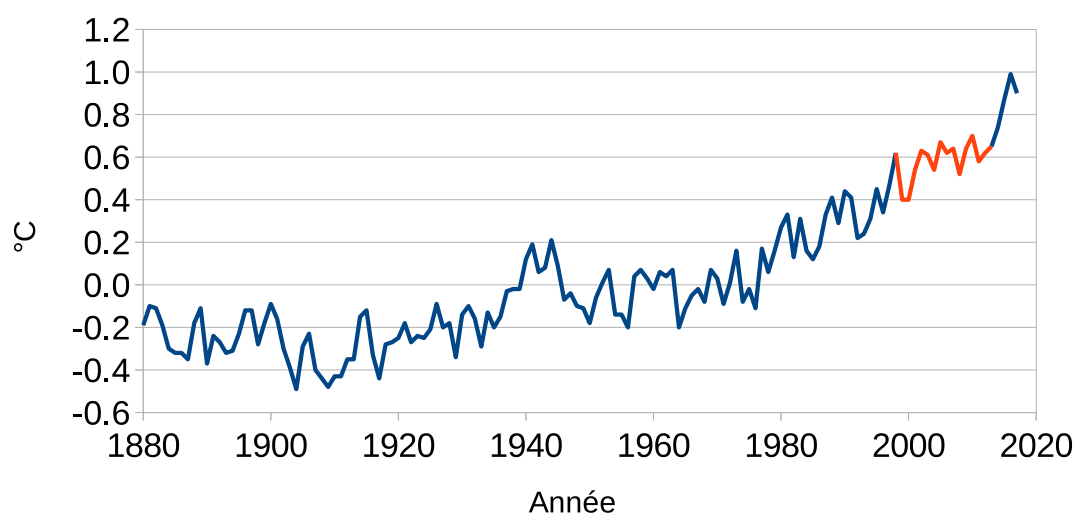

Figure 2. Anomalie moyenne globale des températures de surface : en haut, de 1998 à 2014; en bas, de 1880 à 2018 (d'après les données GISTEMP [38]). La pente de la tendance linéaire indiquée pour 1998-2014 est de 0.097士 $0.041^{\circ} \mathrm{C}$ par décennie.

en été et culmine généralement aux alentours de Noël, d'où le nom du phénomène. Or le réchauffement des eaux de surface sur les côtes est du Pacifique empêche la remontée d'eaux froides profondes. Ainsi, les années El Niño présentent généralement des températures plus élevées que la moyenne. Les autres saisons marquées par des épisodes El Niño depuis 1998 étaient les saisons 2004-5, 2006-7 (El Niño faible) et 200910 (El Niño moyen) - toutes les années qui étaient encore plus chaudes que l'année 1998 étaient donc sous influence El Niño. Partant d'une année El Niño très intense - d'office exceptionnellement chaude - crée donc un biais dans l'estimation de la tendance pendant les années qui suivent. En choisissant les années 1997 ou 1999 comme point de départ, la conclusion aurait été différente, ce qui souligne, s'il y avait encore 

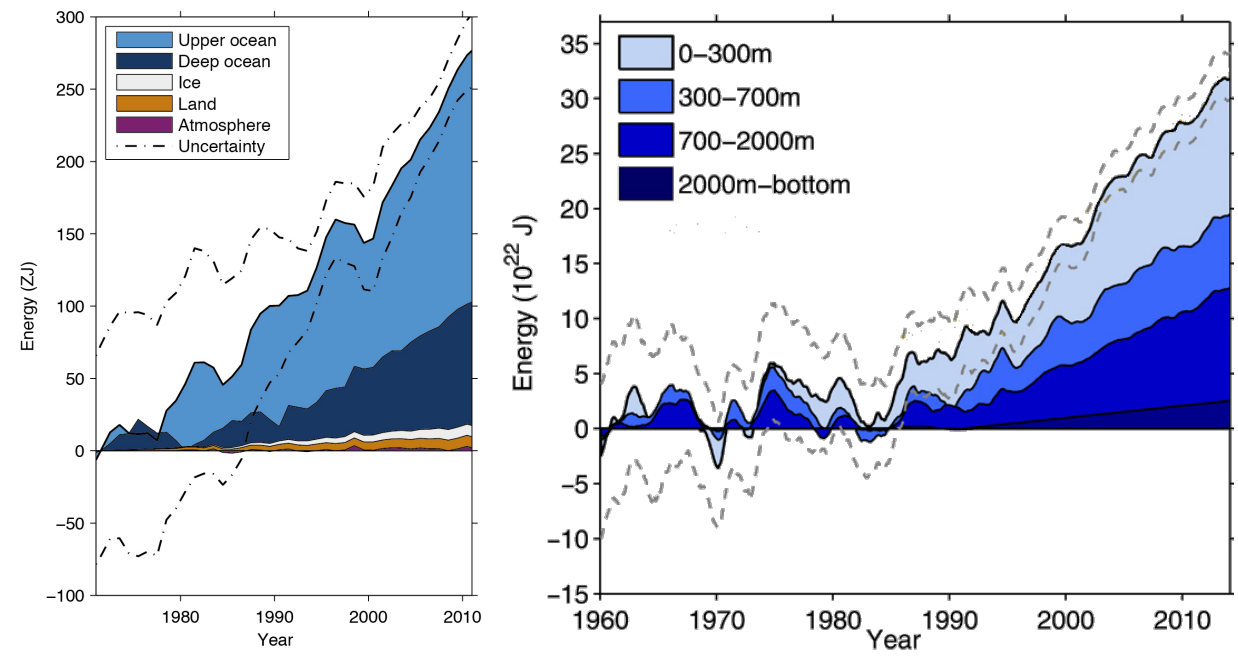

FiguRE 3. Gauche : stockage de chaleur dans le système climatique suivant le $5^{\mathrm{e}}$ Rapport d'évaluation du GIEC [39] ; droite : extension et révision plus récente du stockage dans les océans (adapté de [40]). L'accumulation de chaleur est exprimée par rapport à 1971 (à noter que $\left.1 \mathrm{ZJ}=10^{21} \mathrm{~J}\right)$.

besoin, que l'année 1998 présente un pic de température anormalement élevé. Lorsque nous replaçons cette période climatologiquement courte $^{11}$ (voir le panneau inférieur de la figure 2 dans l'évolution à plus long terme, il ressort clairement que l'augmentation moyenne globale de la température à la surface terrestre au cours de la décennie qui suit 1998 n'est qu'une fluctuation comme tant d'autres que l'on pourrait délimiter dans l'enregistrement.

Reste alors à trouver la raison pour ce ralentissement quand même assez long pendant une période de croissance de $\mathrm{CO}_{2}$ soutenue. Comme mentionné à la section 2, l'évolution de la température à la surface terrestre n'est qu'une des manifestations du réchauffement du système climatique. Pour une vue plus complète, il convient de s'intéresser au bilan d'énergie de ce système, et surtout aux changements de distribution de chaleur. Sur le panneau de gauche de la figure 3, vous pouvez voir l'évolution des contenus thermiques dans les différentes sphères du système climatique de 1971 à 2010 [39]. Le système global est clairement en train d'emmagasiner de la chaleur. Vers la fin de la période d'observation, environ $93 \%$ de la chaleur totale qui s'accumule va dans les océans; $3 \%$ sont consommés sous forme de chaleur latente lors de la fusion des glaces continentales, et $3 \%$ servent à réchauffer les surfaces continentales. Le réchauffement de l'atmosphère ne représente

11. Les caractéristiques climatiques sont généralement définies à partir de données sur 30 ans, et la détermination de tendances climatiques pertinentes demandera donc des intervalles plus longs que cela encore 
que $1 \%$ de la chaleur totale emmagasinée. Des petites fluctuations du taux d'emmagasinage de chaleur par les océans peuvent facilement engendrer des fluctuation marquées dans l'atmosphère. La panneau de droite de la figure 3 présente des résultats d'une analyse et extension plus récente de l'évolution du contenu thermique des océans. Le bilan y est ventilé sur quatre intervalles de profondeur au lieu de deux et la reconstruction remonte plus loin dans le temps. La reconstruction pour les années '60 et '70 fait état d'un contenu thermique marqué par quelques fluctuations, mais sans tendance bien discernable. La tendance à l'augmentation se dégage de manière nette à partir des années '80 et les valeurs sortent définitivement de la bande d'incertitude des estimations des années '60 et '70 dès le milieu des années '90. Depuis l'augmentation se poursuit sans répit significatif.

3.2.3. L'effet de serre n'a jamais été démontré ou prouvé... Face à ce genre d'exigence, la théorie de l'effet de serre ne trouve son pareil que dans le pudding : "The proof of the pudding is in the eating! " adage anglais intraduisible en soi, mais qui a une signification semblable aux expressions « C'est au fruit que l'on connaît l'arbre. » ou « C'est au pied du mur qu'on voit le maçon. » Aucune théorie physique ne peut être « démontrée », ni la mécanique quantique, ni la relativité générale, ni la mécanique newtonienne. Et en fait, aucune de ces théories n'a besoin d'être démontrée ou prouvée; chacune doit plutôt démontrer ou prouver son utilité, nous aider à mieux comprendre comment fonctionne le monde qui nous entoure - bref prouver qu'elle porte des fruits.

La théorie de l'effet de serre découle de principes fondamentaux de la physique. Comme exposé ci-dessus, les propriétés radiatives des gaz comme le dioxyde de carbone, le méthane ou encore de la vapeur d'eau sont connues depuis plus de cent cinquante ans. En appliquant ces propriétés au milieu atmosphérique, on arrive à ce phénomène connu sous le nom d' «effet de serre ». Cette dénomination, choisie au XIX ${ }^{\mathrm{e}}$ siècle par analogie avec l'effet qu'une serre de culture produit par rapport à son environnement, à savoir retenir la chaleur, n'est pas très appropriée et prête souvent à confusion : une serre de culture retient la chaleur en bloquant la convection. L'effet de serre en sciences du climat est par contre lié au transfert radiatif dans dans l'atmosphère, qui ne joue qu'un rôle secondaire pour une serre de culture.

Toutes les observations touchant au bilan d'énergie de l'atmosphère corroborent cette compréhension; à l'heure actuelle aucune ne la contredit, ni sur Terre, ni sur d'autres planètes. Bien au contraire : la théorie de l'effet de serre (aussi mal que son nom puisse-t-avoir été choisi) est la seule théorie physiquement consistante qui permette d'expliquer les observations. En d'autres termes : la théorie de l'effet de serre est aussi bien démontrée que la mécanique quantique, la relativité générale ou encore la théorie de la gravitation. 
Je vous propose dès lors de considérer les résultats suivants provenant de trois études qui traitent du rayonnement sortant de l'atmosphère et qui corroborent la théorie d'une manière particulièrement directe. HARRIES ET AL. 14 présentent pour la première fois une analyse de la différence entre des spectres infrarouges obtenus par deux instruments satellitaires à vingt-sept années d'intervalle : l'INFRARED INTERFEROMETRIC SPECTROMETER (IRIS) de la NASA à bord de Nimbus 4 en 1970 et l'Interferometric Monitor of Greenhouse Gases (IMG) à bord du satellite japonais ADEOS en 1997. Les différences entre les spectres obtenus à l'aide de ces deux instruments montrent une intensification de l'effet de serre en raison des concentrations croissantes de $\mathrm{CH}_{4}, \mathrm{CO}_{2}, \mathrm{O}_{3}, \mathrm{CFC}-11$ et $\mathrm{CFC}-12$ : la température de brillance (brightness temperature) aux longueurs d'onde caractéristiques d'absorption de ces gaz a bien diminué de 1970 à 1997, ce qui montre que les émissions respectives proviennent de couches plus froides de la troposphère, donc plus élevées, en accord avec ce que prédit la théorie de l'effet de serre

Alors que HARRIES ET AL. 14 analysaient la différence entre deux états de l'atmosphère, les deux études de FELDMAN ET AL. [41, 42 ] se basent sur des séries temporelles autrement plus riches en informations. Dans la première de ces deux études, publiée en 2015, FELDMAN ET AL. 41] utilisent des mesures très précises de l'ATMOSPHERIC EMITTED RADIANCE INTERFEROMETER (AERI) prises entre 2000 et 2010, en deux sites différents (à la station Southern Great Plains en OKlahoma et à la station Arctic marine North Slope of Alaska en Alaska). Pendant la période d'observation, le $\mathrm{CO}_{2}$ atmosphérique a augmenté de $22 \mathrm{ppm}$. FELDMAN ET AL. 41] trouvent que cette augmentation a donné lieu à une augmentation de la ré-émission de rayonnement infrarouge depuis l'atmosphère vers la surface à raison de $0,2 \mathrm{~W} / \mathrm{m}^{2}$ par décade aux deux sites (avec une incertitude de l'ordre de $30 \%$ ). Leurs séries temporelles montrent aussi une excellente corrélation des variations saisonnières $d u$ forçage radiatif avec les variations saisonnières $\mathrm{du} \mathrm{CO}_{2}$ dans l'atmosphère.

Dans leur deuxième étude, publiée en 2018, FELDMAN ET AL. 42] se concentrent sur le méthane. Cette fois-ci, ils utilisent des données couvrant les années 2002 à 2012, acquises à la station SouTHERn GREAT PLAINS. Ils trouvent de nouveau une variation du forçage radiatif qui reflète bien l'évolution des concentrations de $\mathrm{CH}_{4}$, saisonnières et à long terme. Depuis l'ère pré-industrielle, la concentration de méthane dans l'atmosphère a augmenté d'environ $720 \mathrm{ppb}$ (parties par milliard en volume, en anglais parts per billion by volume) à plus de $1800 \mathrm{ppb}$ en 2011, soit de $150 \%$ ! Cette augmentation a connu un plateau entre 1999 et 2006, qu'on retrouve bien dans l'évolution du forçage radiatif extraite de la série temporelle de mesures : de 2002 à 2006, on distingue uniquement un cycle saisonnier sur fond constant. À partir de 2007, une 
tendance à l'augmentation de $0.026 \pm 0.006 \mathrm{~W} \mathrm{~m}^{-2} \mathrm{an}^{-1}$ se dégage. $80 \%$ de cette tendance peuvent être attribués directement à l'augmentation de la concentration de $\mathrm{CH}_{4}$, les $20 \%$ restants sont dus à une interaction avec la vapeur d'eau (dans une partie du spectre infrarouge moyen, les bandes d'absorption de $\mathrm{CH}_{4}$ et de $\mathrm{H}_{2} \mathrm{O}$ présentent un recouvrement très important et il n'est pas possible d'isoler les contributions individuelles des deux constituants).

L'universalité de la théorie de l'effet de serre est corroborée par son application à d'autres planètes, comme Vénus (située à 0,7 u.a. ${ }^{12}$ du Soleil). Vénus présente une température assez uniforme de $465^{\circ} \mathrm{C}$ à la surface, à une pression de plus de 90 bar. La surface de Vénus est ainsi plus chaude que celle de Mercure (pourtant situé à seulement 0,4 u.a. du Soleil) : sur Mercure, la température maximale est de $430^{\circ} \mathrm{C}$ et uniquement localement, au point sub-solaire au périhélie. L'explication se trouve dans la composition très particulière de l'atmosphère de Vénus, composée à $96 \%$ de $\mathrm{CO}_{2}$, engendrant un effet de serre très intense.

3.2.4. On ne sait même pas prévoir la météo d'ici cinq jours... Avant de passer aux défis que posent les changements climatiques pour le futur de nos sociétés, il me semble opportun de traiter encore un dernier argument souvent avancé : «Comment peut-on vouloir prédire le climat des prochaines décennies, alors qu'on n'arrive même pas à correctement prédire la météo des cinq jours prochains? 》

C'est une objection qui est, d'un côté infondée, et de l'autre incorrecte, voire dénigrante. De fait, la qualité des prévisions météo a fait d'énormes progrès, surtout au cours des vingt dernières années. BAUER ET AL. [43] ont analysé l'évolution ${ }^{13}$ de la fiabilité des prévisions météorologiques à trois, cinq, sept et dix jours depuis 1981. Les prévisions à trois jours ont amélioré leur taux de fiabilité de quelque $80 \%$ au début des années '80 à environ $98 \%$ en 2014. Pour les prévisions à cinq jours, on est passé d'un peu moins de $60 \%$ à un peu plus de $90 \%$ en même temps. La différence entre les qualités de prévision en hémisphère Sud et en hémisphère Nord s'est rapidement estompée depuis le début des années 2000, en raison de techniques améliorées d'assimilation de données satellitaires. On arrive donc à prédire avec une grande fiabilité la météo des prochains jours.

Enfin, cet argument est sans importance : tout comme la météorologie diffère du climat, les prévisions météorologiques diffèrent des prédictions climatiques. La météorologie décrit l'état local de l'atmosphère sur un court laps de temps, est mesurable en termes de température, précipitations (pluie, neige, ...), vitesse et direction du vent, couverture nuageuse, etc. C'est ce qu'on appelle communément le temps, qui

12. L'unité astronomique est définie comme étant la distance moyenne entre la Terre et le Soleil, $149597870,7 \mathrm{~km}$

13. Le titre de leur article parle même d'une révolution. 
peut changer plusieurs fois par jour en un endroit donné et il peut être différent même à des endroits proches.

Le climat décrit l'évolution annuelle typique des conditions météorologiques. Ses caractéristiques quantitatives sont dérivées des données observationnelles d'un grand nombre d'années (typiquement trente années afin de peupler suffisamment les extrémités de distributions des différentes variables). Le climat ne change que lentement d'habitude. En pratique, la météo détermine, quels vêtements il faut mettre le matin, tandis que le climat détermine quels vêtements il faut avoir dans sa garderobe. Ou, en utilisant les mots d'EDWARD LOREnz 44, le célèbre mathématicien et météorologue américain, qui a découvert de l'effet papillon : «Le climat, c'est ce à quoi vous vous attendez, le temps, c'est ce qui vous arrive $\gg{ }^{14}$

Tout comme les prévisions météorologiques de nos jours, les prédictions climatiques se font à l'aide de modèles numériques. Ces deux types de modèles sont d'un côté très semblables, car basés sur les mêmes lois fondamentales de la physique (mécanique des fluides, thermodynamique, ...). D'un autre côté, les besoins de la prévision météorologique diffèrent profondément de ceux de la prédiction ou projection climatique. Un modèle utilisé à des fins de prévisions météorologiques, part d'un état dit initial, dérivé de données observationnelles, pour en déduire l'évolution sur quelques heures à quelques jours des températures, vitesses de vent, des types et quantités de précipitations et d'autres variables d'intérêt, heure par heure. La qualité des prévisions se dégrade inévitablement au-delà de sept à dix jours puisque l'atmosphère est un système physique présentant des caractéristiques chaotiques. Pendant ce court laps de temps, les conditions aux limites (couvert végétal, température de surface des océans ou encore la circulation océanique) ne vont pas changer de manière significative. Elles seront donc maintenues constantes. Les seules conditions aux limites pour lesquelles on tient compte de variations éventuelles sont l'humidité et la température du sol, ainsi que l'étendue d'une couverture neigeuse, car ces facteurs peuvent varier de manière considérable en quelques heures ou jours. Pour une simulation climatique, ce sont par contre les conditions aux limites qui sont importantes. Il faudra éventuellement même prendre en compte des modifications de la circulation des océans et donc du transport de chaleur impliqué. Idéalement, le modèle de circulation générale atmosphérique sera donc couplé à un modèle de circulation océanique pour répondre à ce besoin. Le couvert végétal peut répondre à l'évolution des conditions climatiques et ainsi modifier les conditions aux limites à travers l'albédo et la rugosité de la surface et de cette

14. "Climate is what you expect, weather is what you get ». D'après ses propres dires, il s'agit d'un vieil aphorisme dont il n'a jamais réussi à retrouver l'origine. Des variantes peuvent en tout cas être retracées jusqu'au XIX ${ }^{\mathrm{e}}$ siècle. Les différences entre climat et météorologie sont donc connues depuis au moins aussi longtemps. 
manière rétroagir sur le climat. Un modèle climatique complet inclura donc aussi un modèle de végétation, dynamique si nécessaire. Pour des périodes d'intérêt plus longues encore (au-delà de plusieurs siècles), d'autres composantes comme des modèles de calottes glaciaires devront être ajoutées.

Une simulation climatique ne fournira pas d'évolution exacte jour après jour des conditions atmosphériques dans un avenir ou un passé lointain. L'évolution calculée de ces conditions sera plutôt utilisée pour établir les statistiques à la base des caractéristiques climatiques typiques (comme, p. ex., température moyenne, minimale ou maximale d'un mois donné de l'année au cours d'une ou de plusieurs décennies). Les modèles climatiques sont évalués de par leur capacité à reproduire l'évolution du climat au cours des 150 dernières années ou alors des conditions climatiques du passé géologique pour lesquels les conditions aux limites peuvent être déterminées avec suffisamment de précision. Des applications à d'autres planètes (comme, p.ex., Vénus) présentant des climats très différents de celui de la Terre permettent aussi d'explorer les limitations d'une implémentation donnée.

\section{Défis : De la STABilisation DU $\mathrm{CO}_{2}$ ATMosphérique AU BUDGET DE CARBONE RESTANT}

En 1988, l'Organisation Météorologique Mondiale (omm) et le Programme des Nations Unies pour L'EnVironnement (PNUE) créent le GROUPE D'EXPERTS INTERGOUVERNEMENTAL SUR L'ÉVOLUTION DU CLIMAT (GIEC), chargé de fournir une évaluation scientifique des risques liés au réchauffement climatique d'origine humaine, des impacts potentiels de ce changement et des stratégies possibles d'adaptation et d'atténuation Le GIEC délivre son premier rapport d'évaluation en 1990 [45], dans lequel il affirme, entre autres

- qu'il est certain que les émissions de gaz à effet de serre (nommément $\mathrm{CO}_{2}, \mathrm{CH}_{4}, \mathrm{CFC}$ et $\mathrm{N}_{2} \mathrm{O}$ ) par l'activité humaine contribuent à une augmentation substantielle des concentrations de ces derniers dans l'atmosphère, ce qui va renforcer l'effet de serre et que la vapeur d'eau, qui est le principal gaz à effet de serre, allait augmenter en réponse à ce renforcement et l'amplifier encore davantage ;

- que ses calculs montrent de manière convaincante, que la moitié de l'augmentation de l'effet se serre détecté est due au $\mathrm{CO}_{2}$ et qu'il est probable que cela allait continuer à être ainsi à l'avenir.

Suite à ce rapport (et un complément publié en 1992), l'ONU prépare la Convention-Cadre des Nations Unies sur les Changements Climatiques (CCNUCC). Elle est adoptée au Sommet de la Terre à Rio de Janeiro en 1992 et précise dans son article 2 que 
«L'objectif de la présente Convention [...] est de stabiliser [...] les concentrations de gaz à effet de serre dans l'atmosphère à un niveau qui empêche toute perturbation anthropique dangereuse du système climatique. »

L'objectif principal de la CCNUCC était donc défini en des termes essentiellement qualitatifs. Aucune information quantitative n'était fournie sur ce que la "perturbation anthropique dangereuse" pourrait englober : un seuil de réchauffement global ou régional à ne pas dépasser? une limitation de l'élévation du niveau marin? limiter la désertification?

Il fallait attendre presque deux décennies (soit $123 \mathrm{GtC}$ d'émissions supplémentaires), avant qu'un premier objectif chiffré ne soit proposé au niveau des NATiOns Unies, dans l'Accord de Copenhague de 2009 (à son article 2) :

« Nous nous accordons à penser qu'une forte diminution des émissions mondiales s'avère indispensable selon les données scientifiques et comme l'a établi le quatrième rapport d'évaluation du GIEC, en vue de réduire ces émissions pour que la hausse de la température de la planète reste inférieure à $2{ }^{\circ} \mathrm{C}$, et entendons prendre, pour atteindre cet objectif, des mesures cadrant avec les données scientifiques et fondées sur l'équité. »

La Conférence des Parties avait refusé d'adopter cet accord-préparé essentiellement par quelques grands états-lors de sa réunion à Copenhague; elle en avait seulement "pris note.' ${ }^{15}$ Ce n'est que l'année suivante, à Cancún, que ce réchauffement moyen de $2{ }^{\circ} \mathrm{C}$ a encore été retenu dans le document final adopté par la conférence.

Enfin, l'Accord de Paris (2015) a confirmé cet objectif de limiter le réchauffement global moyen à $2^{\circ} \mathrm{C}$, tout en l'assortissant d'un cran supplémentaire d'ambition (Article $2, \S 1$ a) :

«Le présent Accord [...] vise à renforcer la riposte mondiale à la menace des changements climatiques, dans le contexte du développement durable et de la lutte contre la pauvreté, notamment en :

a) Contenant l'élévation de la température moyenne de la planète nettement en dessous de $2{ }^{\circ} \mathrm{C}$ par rapport aux niveaux préindustriels et en poursuivant l'action menée pour limiter l'élévation de la température à $1,5^{\circ} \mathrm{C}$ par rapport aux niveaux préindustriels, [...]»

Mais, même avec ces objectifs chiffrés, la mise en œuvre pratique restait difficile, voire impossible : la sensibilité climatique à l'équilibre de 1,5 à $4,5^{\circ} \mathrm{C}$ pour un doublement des concentrations de $\mathrm{CO}_{2}$ dans l'atmosphère par rapport à l'ère pré-industrielle était trop large pour définir

15. C'est par ailleurs la raison pour laquelle cet accord est appelé Copenhagen Accord en Anglais et non pas Copenhagen Agreement. 
une limite de concentration suffisamment bien fondée et acceptable pour des engagements politiques de réduction des émissions.

Une alternative pratique s'est dégagée suite aux analyses publiées quelques mois seulement avant le Sommet de Copenhague (voir, p. ex., [46] pour une revue). Matthews ET AL. [47] ont établi qu'une métrique bien plus robuste liant le réchauffement global aux émissions anthropiques de $\mathrm{CO}_{2}$ serait l'émission cumulée de carbone depuis le début de l'ère industrielle. Ces auteurs montrent que, par Teratonne de carbone émis $\left(10^{12} \mathrm{t}\right.$ soit, par millier de Petagrammes de carbone, $\mathrm{PgC}$ ), la température de l'atmosphère se réchauffe de 1,0 à $2,1^{\circ} \mathrm{C}$.

Ce résultat a servi de base pour établir un budget de carbone restant, c'est-à-dire, une quantité de carbone que l'Humanité peut encore émettre dans l'atmosphère tout en gardant le réchauffement moyen global en-dessous d'un sertain seuil (typiquement 1,5 ou $2^{\circ} \mathrm{C}$ ) avec une probabilité donnée. Un tel budget de carbone était inclus pour le première fois dans le $5^{\mathrm{e}}$ Rapport d'évaluation du GIEC qui date de 2013-2014 [48]. Compte tenu d'autres études semblables à celle de MATTHEWS ET AL. [47, citée ci-dessus, l'évaluation du GIEC adopte une fourchette plus large de 0,8 à $2,5^{\circ} \mathrm{C}$ par millier de $\mathrm{PgC}$ émis.

Le concept comporte quelques faiblesses [48] : dès qu'on tient compte d'autres gaz à effet de serre que le $\mathrm{CO}_{2}$ ou des rétroactions impliquant des émissions de $\mathrm{CO}_{2}$ (comme le dégel des pergélisols, p. ex.), ou encore l'effet des aérosols d'origine anthropique, le lien direct entre émissions cumulées de $\mathrm{CO}_{2}$ et réchauffement se détériore. Le GIEC faisait état d'un budget total disponible de $900 \mathrm{PgC}, 820 \mathrm{PgC}$ et de $790 \mathrm{PgC}$ au départ en 1870 pour limiter le réchauffement global à moins de $2{ }^{\circ} \mathrm{C}$ par rapport à la moyenne de 1861-1880, avec une probabilité supérieure à $33 \%, 50 \%$ et $66 \%$, respectivement. Le budget restant s'obtient en déduisant la quantité totale de carbone déjà émise (estimée à 515 PgC jusqu'en 2011 [48]). Le Rapport de synthèse du $5^{\mathrm{e}}$ Rapport d'évaluation du GIEC inclut aussi des estimations de budget correspondant à la limite de $1,5^{\circ} \mathrm{C}$ [49, Tab. 2.2] : $700 \mathrm{PgC}, 610 \mathrm{PgC}$ et $610 \mathrm{PgC}$ [sic] pour limiter le réchauffement à $1,5^{\circ} \mathrm{C}$ avec une probabilité supérieure à $33 \%, 50 \%$ et $66 \%$, respectivement.

Des complications supplémentaires ont été mises en évidence par la suite. Une première est liée à l'éventuelle mise en œuvre de technologies d'émissions négatives, c'est à dire de séquestration de carbone [50] : les émissions (positives) de $\mathrm{CO}_{2}$ sont plus effectives à augmenter la température que les émissions négatives à la réduire. Les effets ne sont donc plus associatifs dans ce cas. Une autre complication provient de la méthodologie adoptée au départ pour établir ces budgets. Par souci de cohérence, les estimations avaient été basées sur l'évolution des émissions de $\mathrm{CO}_{2}$ déduites de simulations de modèles couplés climat-cycle du carbone et non pas sur des données historiques. En comparaison avec 
ces dernières, les modèles ont tendance à sous-estimer les émissions cumulées de $\mathrm{CO}_{2}$ émis. Le réchauffement simulé, généralement en bon accord avec les observations, est donc attribué à une émission cumulée de $\mathrm{CO}_{2}$ plus faible que celle observée. Millar ET AL. [51] montrent ainsi que l'émission cumulée comptabilisée pour 2015 (545 \pm 75 PgC) n'est atteinte en moyenne par les modèles qu'après 2020, à un moment où le réchauffement modélisé a déjà progressé de $0,3^{\circ} \mathrm{C}$. Au vu de la proximité du réchauffement déjà enregistré jusqu'en 2015 (qu'ils estiment à $0.93 \pm 0.13^{\circ} \mathrm{C}$ ) avec la limite de $1,5^{\circ} \mathrm{C}, 0,3{ }^{\circ} \mathrm{C}$ représente évidemment une marge très importante.

Dans le rapport spécial du GIEC sur le Réchauffement planétaire de $1,5^{\circ} \mathrm{C}$, commandité par la Conférence des Parties réunie à Paris en 2015 et présenté en octobre 2018, ces budgets de carbone ont donc été revus à la hausse. D'après les chiffres fournis [52, Tab. 2.2], on peut calculer que les budgets totaux mentionnés ci-dessus sont de de 210, 160 et $110 \mathrm{PgC}$ plus élevés pour la limite de $1,5^{\circ} \mathrm{C}$, et de 260,200 et $140 \mathrm{PgC}$ pour $2{ }^{\circ} \mathrm{C}$ (respectivement pour une probabilité supérieure à $33 \%, 50 \%$ et $66 \%$ de rester en-dessous de la limite choisie). ${ }^{16} \mathrm{Ce}$ même tableau fournit aussi une évaluation de différentes incertitudes qui affectent les budgets restants pour $1,5^{\circ} \mathrm{C}$, qui restent importantes (plusieurs dizaines à une centaine de $\mathrm{PgC}$ ).

À première vue, le concept du budget de carbone restant est informatif et attractif, car il permet de quantifier le défi que représente la limitation du réchauffement global à 2 , voire $1,5^{\circ} \mathrm{C}$. Cependant, vu les incertitudes et sa dépendance envers des choix stratégiques (comme la mise en ouvre ou non de diverses stratégies plus ou moins agressives d'atténuation), son utilité pratique est devenue de plus en plus discutable avec le temps, d'autant plus que son respect exigerait assez rapidement de procéder à une ventilation par pays.

\section{Conclusions}

L'étude scientifique des principes physiques à la base du changement climatique a été longue et pleine de rebondissements. Les grandes lignes étaient connues dès la fin du XIX ${ }^{\mathrm{e}}$, début du $\mathrm{XX}^{\mathrm{e}}$ siècle.

Une multitude de sources pointent toutes vers une même explication, cohérente et consistante avec toutes les observations : le réchauffement actuel du climat résulte pour la majeure partie de l'augmentation de la concentration de $\mathrm{CO}_{2}$ dans l'atmosphère, suite à l'émission de $\mathrm{CO}_{2}$ par l'activité humaine. $\mathrm{Ce}^{\mathrm{CO}_{2}}$ supplémentaire conduit à un renforcement de l'effet de serre, de manière directe en raison de ses propres caractéristiques d'absorption de rayonnement infrarouge, et pour une

16. À noter que ces chiffres (arrondis à la dizaine) sont approximatifs, car les deux rapports utilisent des périodes de référence légèrement différentes. 
plus grande partie, de manière indirecte par la rétroaction de la vapeur d'eau, le gaz à effet de serre le plus important sur Terre.

Alors que la décision de limiter le réchauffement entérinée dans la CCNUCC date d'il y a presque trente ans déjà, de réelles mesures nécessaires pour avoir la moindre chance de respecter la limitation du réchauffement ne fût-ce qu'à $2^{\circ} \mathrm{C}$ se font toujours attendre. Il ne reste qu'à espérer que cela ne prendra pas autant de temps qu'il a fallu à la théorie de l'effet de serre pour s'établir.

Remerciements. Je remercie LOUIS FRANÇOIS d'avoir partagé avec moi ses connaissances en matière de climat, changement climatique et cycles biogéochimiques. Je tiens aussi à marquer ma profonde reconnaissance et mon respect aux nombreux scientifiques qui compilent et maintiennent à jour les bases de données scientifiques (ici en particulier celles du Goddard Institute for Space Studies (NASA) et du Scripps $\mathrm{CO}_{2}$ Program) et les mettent à disposition du public. Les sites RealClimate, SkePtiCalScience et CARBOnBrief m'ont pour de nombreux sujets fourni des précieux points de départ vers la littérature scientifique.

\section{RÉFÉRENCES}

[1] R. Revelle \& H. E. Suess. Carbon dioxide exchange between atmosphere and ocean and the question of an increase of atmospheric $\mathrm{CO}_{2}$ during the past decades. Tellus, 9(1):18-27, 1957. doi: 10.3402/tellusa.v9i1.9075.

[2] G. N. Plass. The influence of the $15 \mu$ carbon-dioxide band on the atmospheric infra-red cooling rate. Q. J. R. Meteorol. Soc., 82 (353):310-324, 1956. doi: 10.1002/qj.49708235307.

[3] G. N. Plass. The carbon dioxide theory of climatic change. Tellus, 8(2):140-154, 1956. doi: 10.1111/j.2153-3490.1956.tb01206.x.

[4] S. Arrhenius. On the influence of carbonic acid in the air upon the temperature of the ground. The London, Edinburgh, and Dublin Philosophical Magazine and Journal of Science, 41(251):237276, 1896. doi: 10.1080/14786449608620846.

[5] K. Ångström. Ueber die Bedeutung des Wasserdampfes und der Kohlensäure bei der Absorption der Erdatmosphäre. Annalen der Physik, 308(12):720-732, 1900. doi: 10.1002/andp.19003081208.

[6] D. L. Hartmann, A. M. Klein Tank, M. Rusticucci, L. V. Alexander, S. Brönnimann, Y. A.-R. Charabi, F. J. DenTener, E. J. Dlugokencky, D. R. Easterling, A. Kaplan, B. J. Soden, P. W. Thorne, M. Wild, \& P. Zhai. Observations : Atmosphere and surface. In T. F. STOCKER, D. QIn, 
G.-K. Plattner, M. M. B. Tignor, S. K. Allen, J. Boschung, A. Nauels, Y. Xia, V. Bex, \& P. M. Midgley, editors, Climate Change 2013: The Physical Science Basis. Contribution of Working Group I to the Fifth Assessment Report of the Intergovernmental Panel on Climate Change, chapter 2, pages 159254. Cambridge University Press, Cambridge (UK), 2013. ISBN 978-1-107-66182-0. URL https://www.ipcc.ch/site/assets/ uploads/2017/09/WG1AR5_Chapter02_FINAL.pdf.

[7] L. V. Alexander, X. Zhang, T. C. Peterson, J. Caesar, B. Gleason, A. M. G. K. Tank, M. Haylock, D. Collins, B. Tremin, F. Rahimzadeh, A. Tagipour, K. R. Kumar, J. Revadekar, G. Griffithis, L. Vincent, D. B. Stephenson, J. Burn, E. Aguilar, M. Brunet, M. Taylor, M. New, P. Zhai, M. Rusticucci, \& J. L. Vazquez-Aguirre. Global observed changes in daily climate extremes of temperature and precipitation. J. Geophys. Res., 111(D5):D05109, 2006. doi: 10.1029/2005JD006290.

[8] S. B. Fels, J. D. Mahlman, M. D. Schwarzkopf, \& R. W. SINCLAIR. Stratospheric sensitivity to perturbations in ozone and carbon dioxide: Radiative and dynamical response. J. Atmos. Sci., 37(10):2265-2297, 1980. doi: 10.1175/1520-0469(1980)037<2265: $\mathrm{SSTPIO}>2.0 . \mathrm{CO} ; 2$.

[9] B. D. Santer, J. F. Painter, C. Bonfils, C. A. Mears, S. Solomon, T. M. L. Wigley, P. J. Gleckler, G. A. Schmidt, C. Doutriaux, N. P. Gillett, K. E. Taylor, P. W. Thorne, \& F. J. Wentz. Human and natural influences on the changing thermal structure of the atmosphere. Proc. Natl. Acad. Sci. USA, 110(43):17235-17240, 2013. doi: 10.1073/pnas.1305332110.

[10] R. G. Roble \& R. E. Dickinson. How will changes in carbon dioxide and methane modify the mean structure of the mesosphere and thermosphere? Geophys. Res. Lett., 16(12):1441-1444, 1989. doi: 10.1029/GL016i012p01441.

[11] G. Beig, P. Keckhut, R. P. Lowe, R. G. Roble, M. G. Mlynczak, J. Scheer, V. I. Fomichev, D. Offermann, W. J. R. French, M. G. Shepherd, A. I. Semenov, E. E. Remsberg, C. Y. She, F. J. Lübken, J. Bremer, B. R. Clemesha, J. Stegman, F. Sigernes, , \& S. Fadnavis. Review of mesospheric temperature trends. Rev. Geophys., 41(4):1015, 2003. doi: 10.1029/2002RG000121.

[12] J. Laštovička, R. A. Akmaev, G. Beig, J. Bremer, \& J. T. EMmert. Global change in the upper atmosphere. Science, 314: 1253-1254, 2006. doi: 10.1126/science.1135134.

[13] R. Philipona, B. Dürr, C. Marty, A. Ohmura, , \& M. Wild. Radiative forcing - measured at Earth's surface - corroborate the 
increasing greenhouse effect. Geophys. Res. Lett., 31:L03202, 2004. doi: 10.1029/2003GL018765.

[14] J. E. Harries, H. E. Brindley, P. J. Sagoo, \& R. J. BANTGES. Increases in greenhouse forcing inferred from the outgoing longwave radiation spectra of the Earth in 1970 and 1997. Nature, 410:355-357, 2001. doi: 10.1038/35066553.

[15] R. F. Keeling \& S. R. Shertz. Seasonal and interannual variations in atmospheric oxygen and implications for the global carbon cycle. Nature, 358:723-727, 1992. doi: 10.1038/358723a0.

[16] A. C. Manning \& R. F. Keeling. Global oceanic and land biotic carbon sinks from the Sa. c. manning and r. f. keelingcripps atmospheric oxygen flask sampling network. Tellus, 58B(2):95116, 2006. doi: 10.3402/tellusb.v58i2.16803.

[17] S. R. Weart. The Discovery of Global Warming. Harvard University Press, Cambridge (MA), 2 edition, 2008. ISBN 978-0-67403189-0.

[18] H. Le Treut, R. Somerville, U. Cubasch, Y. Ding, C. Mauritzen, A. Mokssit, T. Peterson, \& M. Prather. Historical overview of climate change science. contribution of working group I to the fourth assessment report of the Intergovernmental Panel on Climate Change. In S. Solomon, D. QIn, M. Manning, M. Marquis, K. Averyt, M. M. Tignor, H. L. Miller, JR., \& Z. Chen, editors, Climate Change 2007 - The Physical Science Basis, chapter 1, pages 93-127. Cambridge University Press, Cambridge (UK), 2007. ISBN 78-0-521-705967. URL https://www. ipcc.ch/site/assets/uploads/2018/03/ ar4-wg1-chapter1.pdf.

[19] J. Fourier. Remarques générales sur les températures du globe terrestre et des espaces planétaires. Annales de chimie et de physique, 27:136-167, 1824.

[20] J. Tyndall. The Bakerian Lecture.-on the absorption and radiation of heat by gases and vapours, and on the physical connexion of radiation, absorption, and conduction. Philosophical Transactions, 151, 1861. doi: 10.1098/rstl.1861.0001.

[21] N. EkHolm. On the variations of the climate of the geological and historical past and their causes. Q. J. R. Meteorol. Soc., 27 (117):1-62, 1901. doi: 10.1002/qj.49702711702.

[22] R. T. Pierrehumbert. Principles of Planetary Climate. Cambridge University PRess, Cambridge (UK), 2010.

[23] N. EkHolm. Om klimatets ändringar i geologisk och historisk tid samt deras orsaker. [about climate change in geological and historical times and their causes.]. Ymer, 19(4):353, 1899. Digitized version available from http://runeberg.org/ymer/1899/0377.html.

[24] G. S. Callendar. The artificial production of carbon dioxide and its influence on temperature. Q. J. R. Meteorol. Soc., 64 
(275):223-240, 1938. doi: 10.1002/qj.49706427503.

[25] L. D. KAPlan. The influence of carbon dioxide variations on the atmospheric heat balance. Tellus, 12(2):204-208, 1960. doi: 10.3402/tellusa.v13i2.9444.

[26] F. MÖLLER. On the influence of changes in the $\mathrm{CO}_{2}$ concentration in air on the radiation balance of the Earth's surface and on the climate. J. Geophys. Res., 68(13):3877-3886, 1963. doi: 10.1029/ JZ068i013p03877.

[27] S. Manabe \& R. T. Wetherald. Thermal equilibrium of the atmosphere with a given distribution of relative humidity. J. Atmos. Sci., 24(3):241-259, 1967. doi: 10.1175/1520-0469(1967)024<0241: TEOTAW $>2.0 . \mathrm{CO} ; 2$.

[28] C. D. Keeling. The concentration and isotopic abundances of carbon dioxide in the atmosphere. Tellus, 12(2):200-203, 1960. doi: 10.3402/tellusa.v12i2.9366.

[29] J. Kemp, R. Milne, \& D. S. Reay. Sceptics and deniers of climate change not to be confused. Nature, 464:673, 2010. doi: 10.1038/464673a.

[30] B. NerLich. 'climategate': Paradoxical metaphors and political paralysis. Environmental Values, 19(4):419-442, 2010. doi: 10. $3197 / 096327110 X 531543$.

[31] K. Mulvey, S. Shulman, D. Anderson, N. Cole, J. PiepenBURG, \& J. Sideris. The Climate Deception Dossiers. Union of Concerned Scientists, 2015. URL http://www.ucsusa.org/ decadesofdeception.

[32] J. J. Eberle, H. C. Fricke, J. D. Humphrey, L. Hackett, M. G. Newbrey, \& J. H. Hutchison. Seasonal variability in Arctic temperatures during early Eocene time. Earth Planet. Sci. Lett., 296(3-4):481-486, 2010. doi: 10.1016/j.epsl.2010.06.005.

[33] P. J. Hearty, J. T. Hollin, A. C. Neumann, M. J. O'Leary, \& M. MCCulloch. Global sea-level fluctuations during the Last Interglaciation (MIS 5e). Quat. Sci. Rev., 26(17):2090-2112, 2007. doi: $10.1016 /$ j.quascirev.2007.06.019.

[34] R. Neukom, N. Steiger, J. J. Gómez-Navarro, J. Wang, \& J. P. WeRner. No evidence for globally coherent warm and cold periods over the preindustrial Common Era. Nature, 571:550-554, 2019. doi: 10.1038/s41586-019-1401-2.

[35] R. Neukom, L. A. Barboza, M. P. Erb, F. Shi, J. EmileGeay, M. N. Evans, J. Franke, D. S. Kaufman, L. LüCke, K. Rehfeld, A. Schurer, F. Zhu, S. Brönnimann, G. J. HaKim, B. J. Henley, F. C. Luungqvist, N. MCKay, V. ValLER, \& L. VON GunTEN. Consistent multidecadal variability in global temperature reconstructions and simulations over the common era. Nat. Geosci., 12(8):643-649, 2019. doi: 10.1038/ s41561-019-0400-0. 
[36] S. Brönnimann, J. Franke, S. U. Nussbaumer, H. J. Zumbühl, D. Steiner, M. Trachsel, , G. C. Hegerl, A. Schurer, M. Worni, A. Malik, J. Flückiger, \& C. C. RAIBLE. Last phase of the Little Ice Age forced by volcanic eruptions. Nat. Geosci., 12(8):650-656, 2019. doi: 10.1038/ s41561-019-0402-y.

[37] G. H. Miller, Á. Geirsdóttir, Y. Zhong, D. J. Larsen, B. L. Otto-Bliesner, M. M. Holland, D. A. Bailey, K. A. Refinnider, S. J. Lehman, J. R. Southon, C. Anderson, H. Björnsson, \& T. Thordarson. Abrupt onset of the Little Ice Age triggered by volcanism and sustained by sea-ice/ocean feedbacks. Geophys. Res. Lett., 39(2):L02708, 2012. doi: 10.1029/ 2011 GL050168.

[38] GISTEMP TEAm. GISS surface temperature analysis (GISTEMP), version 3, 2018. Dataset accessed 2018-03-06 at https: //data.giss.nasa.gov/gistemp/.

[39] M. Rhein, S. R. Rintoul, S. Aoki, E. Campos, D. ChamBers, R. A. Feely, S. Gulev, G. C. Johnson, S. A. Josey, A. Kostianoy, C. Mauritzen, D. Roemmich, L. D. Talley, \& F. Wang. Observations : Ocean. In T. F. Stocker, D. Qin, G.-K. Plattner, M. M. B. Tignor, S. K. Allen, J. BoSchung, A. Nauels, Y. Xia, V. Bex, \& P. M. Midgley, editors, Climate Change 2013 : The Physical Science Basis. Contribution of Working Group I to the Fifth Assessment Report of the Intergovernmental Panel on Climate Change, chapter 3, pages 255315. Cambridge University Press, Cambridge (UK), 2013. ISBN 978-1-107-66182-0. URL https://www.ipcc.ch/site/assets/ uploads/2018/02/WG1AR5_Chapter03_FINAL.pdf.

[40] L. Cheng, K. E. Trenberth, J. Fasullo, T. Boyer, J. ABrAhAM, \& J. ZHU. Improved estimates of ocean heat content from 1960 to 2015. Sci. Adv., 3(3):e1601545, 2017. doi: 10.1126/sciadv.1601545.

[41] D. R. Feldman, W. D. Collins, P. J.Gero, M. S. Torn, E. J. Mlawer, \& T. R. ShIPperT. Observational determination of surface radiative forcing by $\mathrm{CO}_{2}$ from 2000 to 2010. Nature, 519:339-343, 2015. doi: 10.1038/nature14240.

[42] D. R. Feldman, W. D. Collins, S. C. Biraud, M. D. RisSer, D. D. Turner, P. J. Gero, J. Tadić, D. Helmig, S. Xie, E. J. Mlawer, T. R. Shippert, \& M. S. Torn. Observationally derived rise in methane surface forcing mediated by water vapour trends. Nat. Geosci., 11(4):238-243, 2018. doi: 10.1038/s41561-018-0085-9.

[43] P. Bauer, A. Thorpe, \& G. Brunet. The quiet revolution of numerical weather prediction. Nature, 525:47, 2015. doi: 10.1038/ nature14956. 
[44] E. N. Lorenz. Climate is what you expect. Unpublished manuscript. URL http://eaps4.mit.edu/research/Lorenz/Climate_ expect.pdf.

[45] IPCC WG I. Climate Change: The IPCC Scientific Asdessment. Cambridge University Press, Cambridge (UK), 1990. ISBN 0-52140720-6. URL https://www .ipcc.ch/report/ar1/wg1/.

[46] J. Rogelu, P. M. Forster, E. Kriegler, C. J. Smith, \& R. SÉFÉRIAN. Estimating and tracking the remaining carbon budget for stringent climate targets. Nature, 571:335-342, 2019. doi: 10.1038/s41586-019-1368-z.

[47] H. D. Matthews, N. P. Gillett, P. A. Stott, \& K. ZickFELD. The proportionality of global warming to cumulative carbon emissions. Nature, 459(7248):829-832, 2009. doi: 10.1038/ nature08047.

[48] M. Collins, R. Knutti, J. Arblaster, J.-L. Dufresne, T. Fichefet, P. Friedlingstein, X. Gao, W. J. Gutowski Jr., T. Johns, G. Krinner, M. Shongwe, C. Tebaldi, A. J. WeAVER, \& M. WeHnER. Long-term climate change: Projections, commitments and irreversibility. In T. F. STOckeR, D. QIN, G.K. Plattner, M. M. B. Tignor, S. K. Allen, J. Boschung, A. Nauels, Y. XiA, V. Bex, \& P. M. Midgley, editors, Climate Change 2013 : The Physical Science Basis. Contribution of Working Group I to the Fifth Assessment Report of the Intergovernmental Panel on Climate Change, chapter 12, pages 10291136. Cambridge University Press, Cambridge (UK), 2013. ISBN 978-1-107-66182-0. URL https://www.ipcc.ch/site/assets/ uploads/2018/02/WG1AR5_Chapter12_FINAL.pdf.

[49] Core Writing Team, R. K. Pachauri, \& L. Meyer, editors. Climate Change 2014. Synthesis Report. IPCC, Geneve (CH), 2014. ISBN 978-92-9169-143-2. URL https://www.ipcc.ch/site/assets/uploads/2018/05/SYR_ AR5_FINAL_full_wcover.pdf.

[50] K. Zickfeld, A. H. MacDougall, \& H. D. Matthews. On the proportionality between global temperature change andcumulative $\mathrm{CO}_{2}$ emissions during periods of net negative $\mathrm{CO}_{2}$ emissions. Environ. Res. Lett., 11:055006, 2016. doi: 10.1088/1748-9326/11/ $5 / 055006$.

[51] R. J. Millar, J. S. Fuglestvedt, P. Friedlingstein, J. RoGelj, M. J. Grubb, H. D. Matthews, R. B. Skeie, P. M. Forster, D. J. Frame, \& M. R. Allen. Emission budgets and pathways consistent with limiting warming to $1.5^{\circ}$ C. Nat. Geosci., 10(10):741-747, 2017. doi: 10.1038/ngeo3031. 
Bulletin de la Société Royale des Sciences, Vol. 88, Actes de colloques, 2019, p. 1 - 30

[52] J. Rogelu, D. Shindell, K. Jiang, S. Fifit, P. Forster, V. Ginzburg, C. Handa, H. Kheshgi, S. KobayaShi, E. Kriegler, L. Mundaca, R. Séférian, \& M. V. ViLARIÑO. Mitigation pathways compatible with $1.5^{\circ} \mathrm{C}$ in the context of sustainable development. In V. MAsson-DelmotTe, P. Zhai, H.-O. Pörtner, D. Roberts, J. Skea, P. R. Shukla, A. Pirani, W. Moufouma-Okia, C. Péan, R. Pidcock, S. Connors, J. B. R. Matthews, Y. Chen, X. Zhou, M. I. Gomis, E. Lonnoy, T. Maycock, M. Tignor, \& T. WaTERFIELD, editors, Global warming of $1.5^{\circ} \mathrm{C}$, chapter 2, pages 93-174. IPCC, 2018. URL https://www.ipcc.ch/site/assets/ uploads/sites/2/2019/05/SR15_Chapter2_High_Res.pdf.

(G. M.)

Guy Munhoven

Laboratoire de Physique Atmosphérique et Planétaire

Institut D'Astrophysique ET DE GÉOPHYSIQUe

Email address: Guy. Munhoven@uliege. be 